\title{
Rectangular-Waveguide Impedance
}

\author{
Dylan F. Williams, Jeff Jargon, Uwe Arz and Paul Hale
}

\begin{abstract}
We discuss the role of the wave impedance in power and temporal measurements and present an approach for accurately estimating the complex wave impedance of the $\mathrm{TE}_{10}$ mode in lossy rectangular waveguide from propagation-constant measurements.
\end{abstract}

Index Terms-Calibration, rectangular waveguide, uncertainty analysis, vector network analyzer, wave impedance.

\section{INTRODUCTION}

$\mathrm{T}$ HE wave impedance of a TE or TM mode in a lossless hollow rectangular waveguide is defined as the ratio of the electric to magnetic field carried by the forward propagating mode in the guide. When the dominant $\mathrm{TE}_{10}$ mode is the only propagating mode in rectangular waveguide, its wave impedance plays a role similar to that of the characteristic impedance in quasi-TEM guides. In this paper, we will estimate the wave impedance of the $\mathrm{TE}_{10}$ mode in a lossy rectangular waveguide from measurements of the propagation constant and discuss the role that impedance plays in power and temporal measurements.

Rectangular-waveguide vector-network-analyzer (VNA) calibrations are designed to measure ratios of the complex amplitudes of forward and backward $\mathrm{TE}_{10}$ modes ("traveling waves" in the nomenclature of [1]) supported by the guides. Most often, these calibrations do not impedance transform the measured scattering parameters to a common constant real reference impedance, as is most often done in on-wafer and coaxial calibrations, but rather implicitly set the reference impedance of these calibrations to the wave impedance (i.e., characteristic impedance) of the $\mathrm{TE}_{10}$ mode. This can be easily verified by noting that most rectangular-waveguide calibrations are based on setting the reflection coefficients of a waveguide section or sliding load to zero.

While the wave impedance reflects the relationship between the electric and magnetic fields of the propagating modes supported in hollow rectangular waveguides, most VNA calibrations do not determine that wave impedance at all. Until recently, these calibrations have been focused on controlling reflections and maximizing power flow, tasks for which the wave impedance is not required.

However, accurately measuring power in rectangular waveguides requires a knowledge of the phase of the wave

Manuscript received March 15, 2015. D. F. Williams, J. Jargon and P. D. Hale are with the National Institute of Standards and Technology, Boulder, CO 80305 USA (phone: 303-497-3138; e-mail: dylan.williams@nist.gov). U. Arz is with the Physikalisch-Technische Bundesanstalt, Bundesallee 100 D38116 Braunschweig Germany. US government publication, not subject to US copyright. impedance [1]. This is because the integral of the Poynting vector over the guide cross section cannot be evaluated without knowing the phase relationships between the electric and magnetic fields there.

Characterizing communications signals in rectangular metal waveguides requires determining not just power flow and reflection coefficients, but also the temporal characteristics of the signal in the guides. For example, we can now manufacture small electro-optic probes with sufficient sensitivity to directly measure the temporal voltage of a modulated signal in a rectangular wave [2] and on-going work promises to develop direct electric-field measurements in rectangular waveguide based on quantum rubidium or cesium standards $[3 ; 4]$.

However, in rectangular waveguide, we cannot identify a single "signal" in the guide, as the magnitude of the wave impedance of the guide varies with frequency, leading to differences in the temporal electric-field and magnetic-field waveforms. For the $\mathrm{TE}_{10}$ mode, for example, the electric field is very large and the magnetic field very small just above cutoff, but as the frequency increases, the electric field falls and magnetic field rises.

In this paper we will develop a practical expression for determining the wave impedance of the $\mathrm{TE}_{10}$ mode in lossy rectangular waveguides from measurements of the propagation constant of the mode, which is easily measured by the thru-reflect-line (TRL) calibration. We will then present recommendations for how to apply the wave impedance in rectangular-waveguide metrology.

\section{LOSSLESS RECTANGULAR WAVEGUIDE}

The modal fields of the dominant $\mathrm{TE}_{10}$ in lossless rectangular waveguide are given by equation 3.107 in [5] as

$$
\begin{gathered}
h_{z}=\cos \left(\frac{\pi x}{a}\right) \\
h_{x}=j \frac{\beta}{k_{\mathrm{c}}^{2}} \frac{\pi}{a} \sin \left(\frac{\pi x}{a}\right)=j \frac{\beta}{k_{\mathrm{c}}} \sin \left(\frac{\pi x}{a}\right) \\
e_{y}=-Z_{\mathrm{h}} \frac{j \beta}{k_{\mathrm{c}}^{2}} \frac{\pi}{a} \sin \left(\frac{\pi x}{a}\right)=-Z_{\mathrm{h}} \frac{j \beta}{k_{\mathrm{c}}} \sin \left(\frac{\pi x}{a}\right),
\end{gathered}
$$

where $a$ is the width of the waveguide, $b$ is the height of the waveguide, $k_{\mathrm{c}}=\pi / a, \beta=\sqrt{k_{0}^{2}-k_{c}^{2}}$ is the propagation factor of the lossless mode, $Z_{\mathrm{h}}=\left(k_{0} / \beta\right) Z_{\text {free-space }}$ is the wave impedance of the $\mathrm{TE}_{10}$ mode, and $k_{0}=\omega / c$ and $Z_{\text {free-space }}=$ $\sqrt{\mu_{0} / \varepsilon_{0}}$ are the wave number and impedance of free space, respectively. The factor $e^{-j \beta z}$ describes the evolution of these lossless modes in the direction of propagation $z$. 


\section{LOSSY RECTANGULAR WAVEGUIDE}

Estimating metal loss at microwave frequencies is usually quite difficult. One of the most effective strategies for estimating the characteristic impedance of lossy printed transmission lines is to use direct measurements of the propagation constant derived from the TRL calibration to estimate the loss of the lines, and then to use these measurements to estimate the characteristic impedance of the lossy lines. In this section we will outline a similar procedure for lossy rectangular waveguide. This will require applying the general waveguide circuit theory of [1], and some of the results of [6], which treats the temporal behavior of the theory's equivalent voltages and currents.

\section{A. Voltage and current description}

To apply the general waveguide circuit theory of $[1 ; 6]$, we start by defining a voltage $v$ and current $i$ that mimic the power and temporal behavior of the electric and magnetic fields in the guide. This simplifies the application of the circuit theory of [1] to the problem. As we will see shortly, there is no loss of generality in doing this.

While a number of definitions for the voltage $v$ and current $i$ are possible, we must keep in mind that different definitions only scale the impedance by a constant frequency-independent factor. We will use a causal power-voltage definition and define the voltage $v$ as the integral of the electric field in the middle of the guide (i.e. integrating over the path at $x=a / 2$ from $y=0$ to $y=b$ ). The power-voltage characteristic impedance $Z_{0}$ of the lossless mode is then given by

$Z_{0}=\frac{\left|v_{0}\right|^{2}}{p_{0}^{*}}=\frac{b^{2} Z_{\mathrm{h}}^{2}\left(\beta / k_{\mathrm{c}}\right)^{2}}{\frac{1}{4} a b\left(\beta / k_{\mathrm{c}}\right)^{2} Z_{\mathrm{h}}}=4 \frac{b}{a} Z_{\mathrm{h}}$,

where $v_{0}=j b Z_{\mathrm{h}} \frac{\beta}{k_{\mathrm{c}}}$ is the voltage obtained by integrating the modal electric field in the middle of the guide at $x=a / 2$ from $y=0$ to $y=b, \quad$ and $p_{0}=\frac{1}{4} a b\left(\frac{\beta}{k_{\mathrm{c}}}\right)^{2} Z_{\mathrm{h}}$ is the integral of the Poynting vector over the guide. Note that $Z_{0}$ is a scaled version of the wave impedance $Z_{\mathrm{h}}$. We will call $Z_{0}$ the characteristic impedance of the lossless guide both to maintain consistency with the nomenclature of $[1 ; 6]$, but also to distinguish it from the wave impedance.

Alternatively, we could set $v_{0}$ equal to $e_{y}$ at some point in the guide (e.g., its center), in which case the voltage would be equal to the total electric field at the center of the guide. These expressions only differ by a fixed frequency-independent constant from the power-current, voltage-current and wave impedances, and do not imply any loss of generality.

\section{B. Separating longitudinal and transverse currents}

Our goal here is to develop formulas for estimating the characteristic impedance $Z$ of the lossy guide from measurements of the propagation constant $\gamma$. We can now apply equations (33)-(36) in [1] for the capacitance $C$, inductance $L$, conductance $G$, and resistance $R$ per unit length of the rectangular waveguide. This will allow us to separate the impacts of the longitudinal and transverse currents in the guide on the loss and characteristic impedance. They are

$$
\begin{gathered}
C=\frac{1}{\left|v_{0}\right|^{2}}\left[\int_{S} \varepsilon^{\prime}\left|e_{\mathrm{t}}\right|^{2} \mathrm{~d} S-\int_{S} \mu^{\prime}\left|h_{z}\right|^{2} \mathrm{~d} S\right] \\
L=\frac{1}{\left|i_{0}\right|^{2}}\left[\int_{S} \mu^{\prime}\left|h_{\mathrm{t}}\right|^{2} \mathrm{~d} S-\int_{S} \varepsilon^{\prime}\left|e_{z}\right|^{2} \mathrm{~d} S\right] \\
G=\frac{\omega}{\left|v_{0}\right|^{2}}\left[\int_{S} \varepsilon^{\prime \prime}\left|e_{\mathrm{t}}\right|^{2} \mathrm{~d} S+\int_{S} \mu^{\prime \prime}\left|h_{z}\right|^{2} \mathrm{~d} S\right] \\
R=\frac{\omega}{\left|i_{0}\right|^{2}}\left[\int_{S} \mu^{\prime \prime}\left|h_{\mathrm{t}}\right|^{2} \mathrm{~d} S+\int_{S} \varepsilon^{\prime \prime}\left|e_{z}\right|^{2} \mathrm{~d} S\right],
\end{gathered}
$$

where the integrals are performed over the cross section of the guide, $i_{0}$ is defined by $v_{0} i_{0}{ }^{*}=p_{0}, \varepsilon=\varepsilon^{\prime}-j \varepsilon^{\prime \prime}$ and $\mu=\mu^{\prime}-$ $j \mu$ ". These expressions ensure that the common relationships in (37) and (38) of [1] between the propagation constant $\gamma$, the characteristic impedance $Z$, and the per-unit-length parameters $R, L, C$, and $G$ hold. That is, that $\gamma=\sqrt{(R+j \omega L)(G+j \omega C)}$ and $Z=\sqrt{(R+j \omega L) /(G+j \omega C)}$.

The expressions in (3) simplify in quasi-TEM lines since $e_{z}$ and $h_{z}$ can be ignored there. In this case, only $h_{\mathrm{t}}$ contributes to the currents in the metal, and adds to $R$ and $L$. When there is no dielectric loss, $C$ becomes constant and $G$ is zero, allowing $Z$ to be accurately estimated from measurements of the propagation constant with $Z=\gamma / j \omega C[7 ; 8]$.

However, in rectangular waveguides, $h_{z}$ cannot be neglected. The magnetic field $h_{z}$ not only contributes to $C$, but currents in the metal due to $h_{z}$ add to $G$, complicating the problem of determining the characteristic impedance of the guide from measurements of $\gamma$. Nevertheless, we can integrate the expressions in (3) and develop an approximation for $Z$ in terms of the measured propagation constant $\gamma$.

The integral of $\varepsilon^{\prime}\left|e_{t}\right|^{2}$ and of $\mu^{\prime}\left|h_{z}\right|^{2}$ in the expression for $C$ and integral of $\mu^{\prime}\left|h_{t}\right|^{2}$ in the expression for $L$ in (3) can be easily evaluated from the fields in (1). The principal contributions to $R$ and $G$ in (3) are from the electric fields in the metal that arise from the currents at the surfaces of metal walls of the rectangular waveguide. When the skin-depth approximation is valid, we can write the current in the metal walls as $J=\left(J_{\mathrm{s}} / \delta\right) e^{-d / \delta}$, where $\delta$ is the skin depth and the surface current density $J_{\mathrm{s}}$ is equal to $\hat{n} \times h$ at the surface of the metal, where $\hat{n}$ is the unit vector normal to the metal surface [5]. We can also approximate the imaginary part of the dielectric constant of the metal as $\varepsilon^{\prime \prime} \cong \sigma / \omega$, where $\sigma$ is the conductivity of the metal. These relations allow the integrals of $\varepsilon^{\prime \prime}|e|^{2}$ in the expressions for $R$ and $G$ to be evaluated from the magnetic field of the lossless guide given in (1).

From these approximations, we obtain

$$
\begin{gathered}
G+j \omega C \cong \frac{1}{2\left|v_{0}\right|^{2}}\left(\frac{1+j}{\sigma \delta}(a+2 b)+j \omega \mu a b \frac{\beta^{2}}{k_{c}^{2}}\right) \\
R+j \omega L \cong \frac{1}{2\left|i_{0}\right|^{2}} \frac{\beta^{2}}{k_{c}^{2}}\left(\frac{1+j}{\sigma \delta} a+j \omega \mu b a\right),
\end{gathered}
$$

where the imaginary part of the term $1+j$ in (4) accounts for the stored magnetic energy in the metal. From (4) we now have 


$$
\frac{R}{\omega L_{0}} \pm \frac{G}{\omega C_{0}}=\frac{R}{\omega L_{0}}\left(1 \pm \frac{a+2 b}{a} \frac{k_{c}^{2}}{\beta^{2}}\right)
$$

where the capacitance $C_{0}$ and the inductance $L_{0}$ of the lossless guide are given by

$$
\begin{aligned}
& C_{0}=\frac{1}{2\left|v_{0}\right|^{2}} \mu a b \frac{\beta^{2}}{k_{c}^{2}} \\
& L_{0}=\frac{1}{2\left|i_{0}\right|^{2}} \mu a b \frac{\beta^{2}}{k_{c}^{2}} .
\end{aligned}
$$

We can now write the propagation constant $\gamma$ and characteristic impedance $Z$ of the dominant mode of the rectangular waveguide as

$$
\begin{gathered}
\gamma=\sqrt{(R+j \omega L)(G+j \omega C)} \\
\approx \sqrt{\left((1+j) R+j \omega L_{0}\right)\left((1+j) G+j \omega C_{0}\right)}
\end{gathered}
$$

and

$$
\begin{gathered}
Z=\sqrt{\frac{R+j \omega L}{G+j \omega C}} \\
\approx \sqrt{\frac{(1+j) R+j \omega L_{0}}{(1+j) G+j \omega C_{0}}} .
\end{gathered}
$$

\section{Approximation for characteristic impedance}

Now we can use measurements of the propagation constant $\gamma$ to approximate $R / \omega L_{0}$ and $G / \omega C_{0}$, and then estimate $Z$. From (8) we see that both the resistive and conductive losses add to the total loss, which gives the approximation

$$
\begin{aligned}
\gamma & \approx j \beta \sqrt{\left(1-j \frac{R}{\omega L_{0}}\right)\left(1-j \frac{G}{\omega C_{0}}\right)} \\
& \approx j \beta\left(1-\frac{1}{2} j\left(\frac{R}{\omega L_{0}}+\frac{G}{\omega C_{0}}\right)\right),
\end{aligned}
$$

or

$$
\frac{R}{\omega L_{0}}+\frac{G}{\omega C_{0}} \approx 2 \frac{\alpha}{\beta}
$$

We can now estimate the characteristic impedance $Z$ of the lossy guide in terms of the dimensions of the guide, the easily calculated characteristic impedance $Z_{0}$ of the lossless mode, and the easily measured propagation constant $\gamma=\alpha+j \beta$ of the lossy mode with

$$
\begin{gathered}
Z=Z_{0}\left(1+\frac{1-j}{2}\left(\frac{R}{\omega L_{0}}-\frac{G}{\omega C_{0}}\right)\right) \\
\approx Z_{0}\left(1+(1-j) \frac{\alpha}{\beta}\left(\frac{1-\frac{a+2 b}{a} \frac{k_{c}^{2}}{\beta^{2}}}{1+\frac{a+2 b}{a} \frac{k_{c}^{2}}{\beta^{2}}}\right)\right) .
\end{gathered}
$$

The formula $\gamma=j k_{0} \sqrt{\varepsilon_{\text {eff }}}$ can be useful in this context. It relates the propagation constant to the effective relative dielectric constant $\varepsilon_{\text {eff }}$ defined in [1] and is often used by VNA calibration software to represent the propagation constant $\gamma$.

\section{Approximation for the wave impedance}

Recognizing that the characteristic impedance $Z$ is just a scaled version of the wave impedance $Z_{\mathrm{h}}$, we also have

$Z_{\mathrm{h}} \approx Z_{\mathrm{h} \text { lossless }}\left(1+(1-j) \frac{\alpha}{\beta}\left(\frac{1-\frac{a+2 b}{a} \frac{k_{c}^{2}}{\beta^{2}}}{1+\frac{a+2 b}{a} \frac{k_{c}^{2}}{\beta^{2}}}\right)\right)$,

where $Z_{\mathrm{h} \text { lossless }}$ is the wave impedance of a lossless section of guide with the same dimensions.

\section{Discussion}

As a general rule, most frequency-point-by-frequency-point rectangular-waveguide circuit designs can be accomplished with the traditional $\mathrm{TE}_{10}$ scattering parameters determined by VNA calibration algorithms that leave the referenceimpedance of rectangular-waveguide calibrations set to the wave impedance of the guide. It would be very disruptive to try to change this industry-wide practice.

Nevertheless, the wave impedance is required for accurate power and temporal waveform measurements in rectangular waveguide [6]. For example, the cross-term in equation (49) of [1] relating the power to wave amplitudes cannot be evaluated without knowing the phase of the characteristic impedance of the guide. Equations (11) and (12) above allow the importance of this term to be evaluated directly.

Figure 1 plots the wave impedance $Z_{\mathrm{h}}$ of the $\mathrm{TE}_{10}$ mode in a lossless rectangular waveguide as a function of the frequency $f$ normalized by the cutoff frequency $f_{\mathrm{c}}$. Clearly the wave impedance varies rapidly near the cutoff frequency and the temporal electric-field and magnetic-field of waveforms with energy concentrated at these frequencies will differ considerably. Figure 2 compares the extreme case of the normalized electric and magnetic fields of five zero-phase tones with energy equally distributed between $1.05 f_{\mathrm{c}}$ and 1.35 $f_{\mathrm{c}}$. Here, the electric and magnetic field waveforms are quite different, as we would expect, given the rise of the wave impedance $Z_{\mathrm{h}}$ near $f_{\mathrm{c}}$.

However, most rectangular waveguide is only used between $1.25 f_{\mathrm{c}}$ and $1.9 f_{\mathrm{c}}$. As a result, the variation of the wave impedance over the band is smaller, making differences in the temporal electric and magnetic-field waveforms difficult to detect. The figure of merit $q=\left(Z_{\mathrm{h}}\left(f_{1}\right)-Z_{\mathrm{h}}\left(f_{2}\right)\right) / Z_{\mathrm{h}}\left(0.5^{*}\left(f_{1}+f_{2}\right)\right)$, where $f_{1}$ and $f_{2}$ are the lower and upper edges of the signal, is a convenient metric for estimating the relative differences in the 


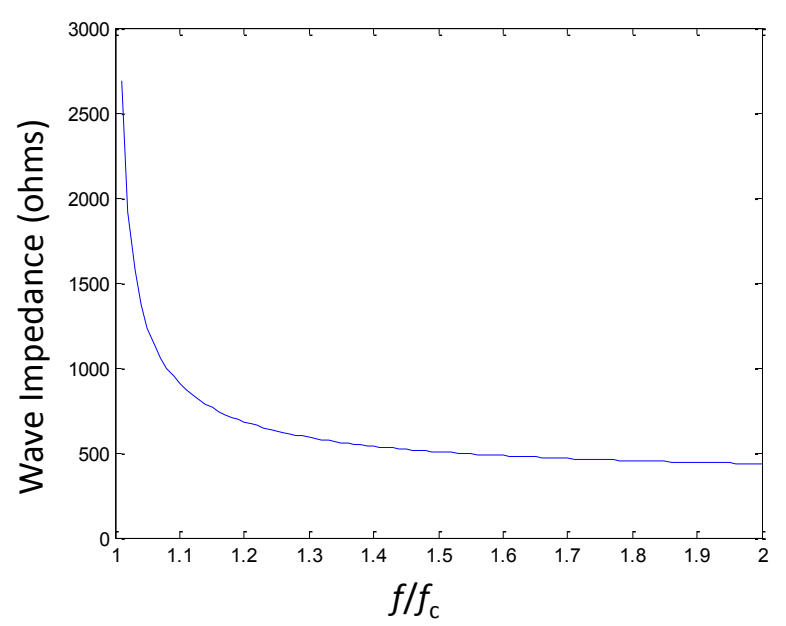

Fig. 1. The characteristic impedance of a uniform section of lossless rectangular waveguide.

electric-field and magnetic-field of waveforms with a relatively small fractional bandwidth. In our extreme example, this ratio was approximately 1 , whereas $q$ is only about 0.05 for a modulated signal with a $10 \%$ bandwidth located near the center of the waveguide band. Thus we would expect that in many practical instances, the differences between the electricfield and magnetic-field waveforms will be small as well.

Nevertheless, even these small differences may be important in precision metrology. In some cases, it may even be important to estimate and correct for the shift of the impedance of the $\mathrm{TE}_{10}$ mode due to waveguide losses.

Here again, the wave impedance or characteristic impedance can be estimated from (11) and (12). Then the normalizing voltage $v_{0}$ or current $i_{0}$ in the theories of $[1 ; 6]$ can be set so that $v$ and $i$ correspond to the desired electric and magnetic field quantities of interest. Now $v$ and $i$ can be calculated from equations (55-56) of [1] with $Z_{\text {ref }}$ set to the characteristic impedance of the guide, which is directly proportional to the wave impedance $Z_{\mathrm{h}}$. If loss is included, (64) of [1] should be applied to transitions to other media. This will correctly relate the powers and temporal behavior $v$ and $i$ to the electric-field and magnetic-field waveforms of the $\mathrm{TE}_{10}$ modes supported by the guides.

The theories of $[1 ; 6]$ can also be used to set the reference impedance of measured scattering parameters in the rectangular-waveguide sections to a constant real value. This can be useful when using software packages based on fixed, real and frequency-independent reference impedances. However, care should be taken in these situations to clearly communicate that a reference-impedance transformation was applied to the measurements, and to clearly specify the value of the resulting reference impedance in the rectangularwaveguide sections.

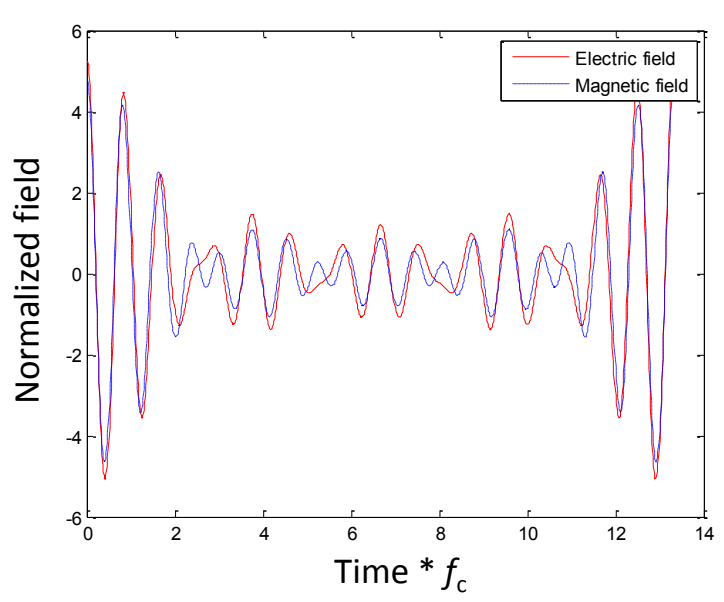

Fig. 2. Normalized electric and magnetic fields of a set of five zero-phase tones with equal power in a uniform section of lossless rectangular waveguide.

\section{REFERENCES}

[1] R. B. Marks and D. F. Williams, "A general waveguide circuit theory," J. Res. Nat. Instit. Standards and Technol., vol. 97, no. 5, pp. 533-562, Sept.1992.

[2] D. J. Lee, J. Y. Kwon, Y. P. Hong, and J. A. Jargon, "Photonic-Assisted Endoscopic Analysis of W-Band Waveguide," IEEE Photonics Conference, pp. 216217, 2014.

[3] C. L. Holloway, J. A. Gordon, A. Schwarzkopf, D. A. Anderson, S. A. Miller, N. Thaicharoen, and G. Raithel, "Sub-wavelength imaging and field mapping via electromagnetically induced transparency and Autler-Townes splitting in Rydberg atoms," Applied Physics Letters, vol. 104, no. 244102, pp. 1-5, 2014.

[4] C. L. Holloway, J. A. Gordon, S. Jefferts, A. Schwarzkopf, D. A. Anderson, S. A. Miller, N. Thaicharoen, and G. Raithel, "Broadband Rydberg Atom-Based Electric-Field Probe for SI-Traceable, Self-Calibrated Measurements," Antennas and Propagation, IEEE Transactions on, vol. 62, no. 12, pp. 6169-6182, Dec.2014.

[5] R. E. Collin, Foundations for Microwave Engineering McGraw-Hill, Inc., 1966.

[6] D. F. Williams and B. Alpert, "Causality and waveguide circuit theory," IEEE Trans. Microwave Theory Tech., vol. 49, no. 4, pp. 615-623, Apr.2001.

[7] R. B. Marks and D. F. Williams, "Characteristic Impedance Determination using Propagation Constant Measurement," IEEE Microwave and 
Guided Wave Letters, vol. 1, no. 6, pp. 141-143, June1991.

[8] D. F. Williams and R. B. Marks, "Transmission Line Capacitance Measurement," IEEE Microwave and Guided Wave Letters, vol. 1, no. 9, pp. 243-245, Sept.1991. 